ANNALES

POLONICI MATHEMATICI

$80(2003)$

\title{
Robin functions and extremal functions
}

\author{
by T. Bloom (Toronto), N. Levenberg (Auckland) \\ and S. MA'U (Auckland)
}

$$
\begin{aligned}
& \text { Abstract. Given a compact set } K \subset \mathbb{C}^{N} \text {, for each positive integer } n \text {, let } \\
& \begin{aligned}
V^{(n)}(z) & =V_{K}^{(n)}(z) \\
& :=\sup \left\{\frac{1}{\operatorname{deg} p} V_{p(K)}(p(z)): p \text { holomorphic polynomial, } 1 \leq \operatorname{deg} p \leq n\right\} .
\end{aligned}
\end{aligned}
$$

These "extremal-like" functions $V_{K}^{(n)}$ are essentially one-variable in nature and always increase to the "true" several-variable (Siciak) extremal function,

$$
V_{K}(z):=\max \left[0, \sup \left\{\frac{1}{\operatorname{deg} p} \log |p(z)|: p \text { holomorphic polynomial, }\|p\|_{K} \leq 1\right\}\right] .
$$

Our main result is that if $K$ is regular, then all of the functions $V_{K}^{(n)}$ are continuous; and their associated Robin functions

$$
\varrho_{V_{K}^{(n)}}(z):=\limsup _{|\lambda| \rightarrow \infty}\left[V_{K}^{(n)}(\lambda z)-\log (|\lambda|)\right]
$$

increase to $\varrho_{K}:=\varrho_{V_{K}}$ for all $z$ outside a pluripolar set.

\section{Introduction. Let}

$$
L:=\left\{u \text { plurisubharmonic }(\mathrm{psh}) \text { in } \mathbb{C}^{N}: u(z) \leq \log ^{+}|z|+C\right\}
$$

denote the class of psh functions of logarithmic growth on $\mathbb{C}^{N}$ (here $|z|=$ $\left(\sum_{i=1}^{N}\left|z_{i}\right|^{2}\right)^{1 / 2} ; \log ^{+}|z|=\max (0, \log |z|)$; and the constant $C$ can depend on $u$ ). We also consider the class

$$
L^{+}:=\left\{u \in L: \log ^{+}|z|+C_{1} \leq u(z) \leq \log ^{+}|z|+C_{2} \text { for some } C_{1}, C_{2}\right\} .
$$

These classes arise naturally in complex potential theory in $\mathbb{C}$ and in pluripotential theory in $\mathbb{C}^{N}$. For a bounded Borel set $E$ in $\mathbb{C}^{N}$, define

$$
V_{E}(z):=\sup \{u(z): u \in L, u \leq 0 \text { on } E\} .
$$

2000 Mathematics Subject Classification: 31C15, 32F05.

Key words and phrases: plurisubharmonic, extremal function, Robin function. 
The upper semicontinuous (usc) regularization $V_{E}^{*}(z):=\lim \sup _{\zeta \rightarrow z} V_{E}(\zeta)$ is called the (Siciak) extremal function of $E$. It is well known that $V_{E}^{*} \in L^{+}$ if and only if $E$ is nonpluripolar; i.e., if $u$ psh is $-\infty$ on $E$, then $u \equiv-\infty$. If $K$ is a compact set in $\mathbb{C}^{N}$, then the extremal function in (0.1) can be obtained via the formula

$$
V_{K}(z)
$$

$:=\max \left[0, \sup \left\{\frac{1}{\operatorname{deg} p} \log |p(z)|: p\right.\right.$ holomorphic polynomial, $\left.\left.\|p\|_{K} \leq 1\right\}\right]$

([K, Theorem 5.1.7]). Here, $\|p\|_{K}:=\sup _{z \in K}|p(z)|$ denotes the uniform norm on $K$.

To study the asymptotic behavior of such functions, we recall the notion of the Robin function associated to a function $u \in L$. First of all, suppose that $K \subset \mathbb{C}^{N}$ is compact and regular, i.e., $V_{K}=V_{K}^{*}$ (equivalently, $V_{K}$ is continuous). The Robin function of $K$ is $\varrho_{K}: \mathbb{C}^{N} \rightarrow \mathbb{R} \cup\{-\infty\}$ defined by

$$
\varrho_{K}(z):=\limsup _{|\lambda| \rightarrow \infty}\left[V_{K}(\lambda z)-\log (|\lambda|)\right] .
$$

More generally, for $u: \mathbb{C}^{N} \rightarrow \mathbb{R}$ in $L$ we define the Robin function of $u$ to be

$$
\varrho_{u}(z):=\limsup _{|\lambda| \rightarrow \infty}[u(\lambda z)-\log (|\lambda|)]
$$

(hence $\left.\varrho_{K}=\varrho_{V_{K}}\right)$. Note that for $\lambda \in \mathbb{C}, \varrho_{u}(\lambda z)=\log |\lambda|+\varrho_{u}(z)$ (logarithmic homogeneity; cf. Section 5). It is known [Bl] that for $u \in L$, the Robin function $\varrho_{u}(z)$ is plurisubharmonic in $\mathbb{C}^{N}$; indeed, either $\varrho_{u} \in L$ or $\varrho_{u} \equiv$ $-\infty$.

Our aim in this note is two-fold: first, we discuss the Robin function $\varrho_{u}(z)$-more precisely, the Robin constant - associated to a function $u \in L$ in one complex variable. Using these results, we then analyze the Robin function associated to certain "extremal-like" functions associated to a compact set $K \subset \mathbb{C}^{N}, N>1$. For each positive integer $n$, let

$$
\begin{aligned}
& V^{(n)}(z)=V_{K}^{(n)}(z) \\
& \quad:=\sup \left\{\frac{1}{\operatorname{deg} p} V_{p(K)}(p(z)): p \text { holomorphic polynomial, } 1 \leq \operatorname{deg} p \leq n\right\} .
\end{aligned}
$$

These functions $V_{K}^{(n)}$ (discussed in [BCL]) are essentially one-variable in nature and always increase to the "true" extremal function, $V_{K}$. Our main result is that if $K$ is regular, then all of the functions $V_{K}^{(n)}$ are continuous. Concerning their associated Robin functions $\varrho_{V_{K}^{(n)}}$, we show that $\varrho_{V_{K}^{(1)}}$ is also continuous and, in this case, the limsup in (0.3) can be replaced by 
limit; i.e., the limit exists. Moreover,

$$
\lim _{n \rightarrow \infty} \varrho_{V^{(n)}}(z)=\varrho_{K}(z)
$$

for q.e. $z \in \mathbb{C}^{N}$ (i.e., all $z$ outside a pluripolar set). We mention that (0.4) is not an immediate consequence of the monotone convergence of the functions $V_{K}^{(n)}$ to the function $V_{K}$; indeed, a necessary and sufficient condition for (0.4) to hold involves the Monge-Ampère measures of these functions (cf. [BT]); this condition is usually difficult to verify. We end with some open questions related to the notions in this and the [BCL] paper.

We would like to thank the referee for an extremely careful reading of the manuscript and many helpful comments. Also, we are grateful to Professor J. Siciak for some useful remarks which we have incorporated in the text.

1. Subharmonic functions in $\mathbb{C}$. In this section, we work exclusively in $\mathbb{C}$. The major question we want to address is the following: for which functions $u \in L$ does the limit

$$
\lim _{|t| \rightarrow \infty}[u(t)-\log |t|]
$$

exist? We first discuss some known results about subharmonic functions in $\mathbb{C}$. Let $\mu$ be a nonnegative Borel measure on $\mathbb{C}$ of finite total mass. Under what conditions is $\mu$ the Laplacian of a function in $L$ ?

Proposition 1.1. Suppose that $\int_{|t| \leq 1} \log |t| d \mu(t)>-\infty$. Then $\mu(1):=$ $\int_{t \in \mathbb{C}} d \mu(t) \leq 1$ if and only if

$$
u(z):=\int_{t \in \mathbb{C}}[\log |z-t|-\log |t|] d \mu(t)
$$

belongs to $L$.

Remark. From Brelot's theorem (cf. [R]), it follows that if $\mu(1)<\infty$ and

$$
\int_{|t| \leq 1} \log |t| d \mu(t)>-\infty
$$

then

$$
u(z):=\int_{t \in \mathbb{C}}[\log |z-t|-\log |t|] d \mu(t)
$$

is a subharmonic (shm) function in $\mathbb{C}$.

Proof of Proposition 1.1. Introduce the notation $n(r):=\int_{|t| \leq r} d \mu(t)$. We first recall Jensen's formula: let $u$ be shm in the disk $\{z:|z|<R\}$ and 
harmonic in a neighborhood of the origin. Then for any $r<R$,

$$
\begin{aligned}
M_{u}(r) & :=\frac{1}{2 \pi} \int_{0}^{2 \pi} u\left(r e^{i \theta}\right) d \theta=u(0)+\int_{0}^{r} \frac{n(t)}{t} d t \\
& =u(0)+\int_{0}^{r}[\log r-\log |t|] d n(t) .
\end{aligned}
$$

Suppose $u$ as in (1.1) is in $L$ but $\mu(1)=\alpha>1$. Without loss of generality we may assume $u(0)=0$. Since $u \in L$,

$$
\lim _{r \rightarrow \infty}\left[M_{u}(r)-\log r\right]<\infty .
$$

Fix $0<\beta<1-1 / \alpha$. Then, since $\lim _{t \rightarrow \infty} n(t)=\alpha$, there exists $r_{0}>1$ such that for all $r>r_{0}$,

$$
(1-\beta) n\left(r^{\beta}\right)=: 1+\delta>1 .
$$

But

$$
\begin{aligned}
M_{u}(r)-\log r & =\int_{0}^{r}[\log r-\log |t|] d n(t)-\log r \\
& \geq \int_{0}^{r^{\beta}}[\log r-\log |t|] d n(t)-\log r \\
& \geq(1-\beta)(\log r) n\left(r^{\beta}\right)-\log r=\delta \log r,
\end{aligned}
$$

which contradicts (1.3).

For the converse, we may assume $\mu(1)=1$. We want to find a constant $C$ such that

$$
u(z):=\int_{t \in \mathbb{C}}[\log |z-t|-\log |t|] d \mu(t) \leq C+\log |z|
$$

for all $|z| \geq 1$. Fix such a $z$ and write

$$
\begin{aligned}
u(z)= & \int_{|t| \leq 1} \log \left|1-\frac{z}{t}\right| d \mu(t) \\
& +\int_{|t|>1,|z|<|t|} \log \left|1-\frac{z}{t}\right| d \mu(t)+\int_{|t|>1,|z| \geq|t|} \log \left|1-\frac{z}{t}\right| d \mu(t) \\
\leq & {\left[n(1) \log |z|+c_{1}\right]+[(1-n(1)) \log 2]+\int_{|t|>1,|z| \geq|t|} \log \frac{2|z|}{|t|} d \mu(t) } \\
\leq & {\left[n(1) \log |z|+c_{1}\right]+[(1-n(1)) \log 2]+\int_{|t|>1} \log 2|z| d \mu(t) } \\
= & {\left[n(1) \log |z|+c_{1}\right]+[(1-n(1)) \log 2]+[1-n(1)] \log 2|z| } \\
= & \log |z|+C .
\end{aligned}
$$


It follows from Proposition 1.1 that if $u \in L$ and $u(0)=0$ then $u$ can be written as in (1.1) with

$$
d \mu(t)=\frac{-1}{4 \pi i} \Delta u(t) d t \wedge d \bar{t} \quad \text { and } \quad \mu(1) \leq 1 .
$$

The fact that $\mu(1) \leq 1$ for $u \in L$ follows easily from Jensen's formula (1.2): if $\mu(1)>1$, then there exist $\delta>0$ and $r_{0}$ such that for all $r>r_{0}$, we have $n(r) \geq 1+\delta$. Fixing such an $r$, we obtain

$$
M_{u}(r)-M_{u}\left(r_{0}\right)=\int_{r_{0}}^{r} \frac{n(t)}{t} d t \geq(1+\delta) \log \frac{r}{r_{0}} .
$$

The left-hand side of this inequality is dominated by $\log (1+r)$ plus a constant - for all $r$-yielding a contradiction.

To show that the problem described in (1.0) is nontrivial, we begin with an explicit example of a continuous function $u \in L^{+}(\mathbb{C})$ for which the limit (1.0) does not exist.

Proposition 1.2. There exists $u \in L^{+}(\mathbb{C}) \cap C(\mathbb{C})$ for which $u(t)-\log |t|$ does not have a limit as $|t| \rightarrow \infty$.

Proof. The idea is to construct a sequence of continuous subharmonic functions

$$
u_{j}(t):=\log ^{+} \frac{\left|t-t_{j}\right|}{r_{j}}+\log r_{j}
$$

in $L^{+}(\mathbb{C})$ having Laplacians supported on circles $\left|t-t_{j}\right|=r_{j}$ of smaller and smaller radii $r_{j}$ with centers $t_{j}$ marching to infinity in such a way that an infinite sum

$$
u(t):=\sum_{j} \varepsilon_{j} u_{j}(t)
$$

gives us the desired function. To make this precise, we first choose a sequence $\left\{t_{j}\right\}$ of positive numbers with $t_{1}>2$ and $t_{j} \uparrow \infty$ and with

$$
2 t_{j} \leq t_{j+1} \leq 4 t_{j}
$$

for all $j$. Next, choose a sequence $\left\{\varepsilon_{j}\right\}$ of positive numbers with

$$
\varepsilon_{j} \downarrow 0, \quad \sum_{j} \varepsilon_{j}=1, \quad \sum_{j} \varepsilon_{j} \log t_{j}<\infty .
$$

Finally, define the sequence $\left\{r_{j}\right\}$ of positive numbers by

$$
r_{k}:=1 /\left[\prod_{j=1}^{k-1} t_{j}^{\varepsilon_{j} / \varepsilon_{k}}\right] .
$$


Note that by the choice of $r_{k}$ in (1.6), we have

Now let

$$
\varepsilon_{k} \log \frac{t_{k}}{r_{k}}=\sum_{j=1}^{k} \varepsilon_{j} \log t_{j}
$$

$$
u_{j}(t):=\log +\frac{\left|t-t_{j}\right|}{r_{j}}+\log r_{j} \quad \text { and } \quad u(t):=\sum_{j} \varepsilon_{j} u_{j}(t)
$$

as above. We show this $u$ satisfies the conditions stated in the proposition.

(i) $u$ is in $L^{+}(\mathbb{C})$. If $t_{k} \leq|t| \leq t_{k+1}$, then

Hence

$$
\left|t-t_{j}\right| \leq|t|+t_{j} \leq \begin{cases}2|t| & \text { for } j \leq k \\ 2 t_{j} & \text { for } j>k\end{cases}
$$

$$
u(t) \leq \sum_{j \leq k} \varepsilon_{j} \log 2|t|+\sum_{j>k} \varepsilon_{j} \log 2 t_{j} \leq\left[\log 2+\sum_{j} \varepsilon_{j} \log t_{j}\right]+\log |t|
$$

by (1.5). In the other direction, if we write

$$
u(t)=\sum_{j \neq k, k+1} \varepsilon_{j} \log |t|+\sum_{j \neq k, k+1} \varepsilon_{j} \log \frac{\left|t-t_{j}\right|}{|t|}+\varepsilon_{k} u_{k}(t)+\varepsilon_{k+1} u_{k+1}(t)
$$

and we use the estimates

$$
\begin{gathered}
\frac{\left|t-t_{j}\right|}{|t|}>\frac{|t|-t_{j}}{|t|}>\frac{1}{2} \quad \text { for } j<k \quad \text { since }|t| \geq t_{k}>2 t_{j}, \\
\frac{\left|t-t_{j}\right|}{|t|}>\frac{t_{j}-|t|}{|t|}>\frac{1}{2} \quad \text { for } j>k+1 \quad \text { since } t_{j} \geq 2 t_{k+1}>2|t| ;
\end{gathered}
$$

we obtain

$$
\begin{aligned}
u(t) & \geq \sum_{j \neq k, k+1} \varepsilon_{j} \log |t|+\log 1 / 2+\varepsilon_{k} u_{k}(t)+\varepsilon_{k+1} u_{k+1}(t) \\
& \geq \log |t|+\log \frac{1}{2}+\varepsilon_{k} \log \frac{r_{k}}{|t|}+\varepsilon_{k+1} \log \frac{r_{k+1}}{|t|} \\
& \geq \log |t|+\log \frac{1}{2}+\varepsilon_{k} \log \frac{r_{k}}{4 t_{k}}+\varepsilon_{k+1} \log \frac{r_{k+1}}{t_{k+1}} \quad(\text { from } \\
& \geq \log |t|+\log \frac{1}{2}-\varepsilon_{k} \log 4-2 \varepsilon_{k+1} \log \frac{t_{k+1}}{r_{k+1}} \\
& \geq \log |t|-\log 8-2 \sum_{j=1}^{k+1} \varepsilon_{j} \log t_{j} \\
& \geq \log |t|-\log 8-2 \sum_{j=1}^{\infty} \varepsilon_{j} \log t_{j}=\log |t|+c_{1},
\end{aligned}
$$

where the last two lines follow from (1.5) and (1.7). 
(ii) There exists $\delta>0$ with $\liminf _{k \rightarrow \infty}\left[u\left(-t_{k}\right)-u\left(t_{k}\right)\right] \geq \delta$. For

$$
\begin{aligned}
u\left(-t_{k}\right)-u\left(t_{k}\right) & >\varepsilon_{k}\left[u_{k}\left(-t_{k}\right)-u_{k}\left(t_{k}\right)\right]=\varepsilon_{k} \log \frac{2 t_{k}}{r_{k}} \\
& \left.>\varepsilon_{k} \log \frac{t_{k}}{r_{k}}=\sum_{j=1}^{k} \varepsilon_{j} \log t_{j} \quad \text { (from }(1.7)\right)
\end{aligned}
$$

and the result follows from convergence of $\sum_{j=1}^{\infty} \varepsilon_{j} \log t_{j}$ (see (1.5)).

(iii) $u$ is continuous on $\mathbb{C}$. It suffices to show that the series $\sum_{j} \varepsilon_{j} u_{j}$ converges uniformly on compact sets in $\mathbb{C}$ since each $u_{j}$ is continuous on $\mathbb{C}$. Fix $t \in \mathbb{C}$. If there exists $r>0$ so that the disk $\Delta(t, r):=\{z:|z-t|<r\}$ avoids each of the disks $\bar{\Delta}\left(t_{j}, r_{j}\right)$, then clearly the series $\sum_{j} \varepsilon_{j} u_{j}$ converges uniformly to $u$ on $\bar{\Delta}(t, r / 2)$. Otherwise we can choose $r>0$ sufficiently small so that the disk $\Delta(t, r):=\{z:|z-t|<r\}$ meets at most one of the disks $\bar{\Delta}\left(t_{j}, r_{j}\right)$, say $\Delta(t, r) \cap \Delta\left(t_{k}, r_{k}\right) \neq \emptyset$. Then for $z \in \Delta(t, r)$,

$$
u(z)=\varepsilon_{k} \log r_{k}+\sum_{j=1, j \neq k}^{\infty} \varepsilon_{j} \log \left|z-t_{j}\right| \quad \text { if } z \in \Delta(t, r) \cap \Delta\left(t_{k}, r_{k}\right),
$$

while

$$
u(z)=\sum_{j=1}^{\infty} \varepsilon_{j} \log \left|z-t_{j}\right| \quad \text { if } z \in \Delta(t, r) \backslash \Delta\left(t_{k}, r_{k}\right) .
$$

Note that $\left|t-t_{j}\right|>1$ for $j>k$; thus, for $z \in \Delta(t, r)$, we also have $\left|z-t_{j}\right|>1$; hence if $N>k$ we obtain the estimate

$$
\begin{aligned}
\mid u(z) & -\sum_{j=1}^{N} \varepsilon_{j} u_{j}(z)\left|=\sum_{j=N+1}^{\infty} \varepsilon_{j} \log \right| z-t_{j} \mid \leq \sum_{j=N+1}^{\infty} \varepsilon_{j} \log \left(r+\left|t-t_{j}\right|\right) \\
& \leq \sum_{j=N+1}^{\infty} \varepsilon_{j} \log M\left|t-t_{j}\right|=\log M \sum_{j=N+1}^{\infty} \varepsilon_{j}+\sum_{j=N+1}^{\infty} \varepsilon_{j} \log \left|t-t_{j}\right|
\end{aligned}
$$

where $M=M(r)$. Thus given $\varepsilon>0$, we choose $N>k$ sufficiently large so that

$$
\sum_{j=N+1}^{\infty} \varepsilon_{j}<\frac{\varepsilon}{2 \log M} \text { and } \sum_{j=N+1}^{\infty} \varepsilon_{j} \log \left|t-t_{j}\right|<\frac{\varepsilon}{2} .
$$

This yields

$$
\left|u(z)-\sum_{j=1}^{N} \varepsilon_{j} u_{j}(z)\right|<\varepsilon
$$

for all $z \in \Delta(t, r)$; i.e., the partial sums $u_{N}(z):=\sum_{j=1}^{N} \varepsilon_{j} u_{j}(z)$ converge uniformly to $u(z)$ on $\Delta(t, r)$. 
REMARK. Siciak has pointed out how to construct lots of examples using facts from complex potential theory: start with a compact, nonpolar, polynomially convex set $K \subset \mathbb{C}$ such that $0 \in K$ is the only irregular point of $K$. Then the extremal function $V_{K}^{*}$ belongs to $L^{+}(\mathbb{C})$ and is continuous on $\mathbb{C} \backslash\{0\}$. The function $u(z):=V_{K}^{*}(1 / z)+\log |z|$ for $z \neq 0$ extends continuously to $z=0$ upon setting $u(0):=\lim _{z \rightarrow 0, z \neq 0} u(z)=-\log$ cap $K$, where cap $K$ denotes the logarithmic capacity of $K$, and this $u$ provides another example of a function satisfying the criteria of Proposition 1.2. As a concrete example of such a set $K$, take $K:=\{0\} \cup \bigcup_{k=1}^{\infty}\left[e^{-2 \cdot 3^{k}}, e^{-3^{k}}\right]$.

We still recover a one-sided estimate for general functions $u \in L^{+}(\mathbb{C})$. We claim that we may write $u$ as the sum of the logarithmic potential of its Laplacian plus a constant:

$$
u(t):=\int \log |t-s| d \mu(s)+\left[u(0)-\int \log |s| d \mu(s)\right]
$$

where

$$
d \mu(t)=\frac{-1}{4 \pi i} \Delta u(t) d t \wedge d \bar{t}
$$

is the probability measure associated to the Laplacian $\Delta u(t)$. Recall that we defined

$$
n(r):=\int_{|t| \leq r} d \mu(t)
$$

for $r>0$. Since we are only concerned with asymptotic behavior of $u$, we may assume there exists $\delta>0$ with $n(r)=0$ for $r \leq \delta$. The following facts follow from arguments similar to those used in Proposition 1.1:

(i) $\int \log |s| d \mu(s)=\int \log r d n(r)$ is finite;

(ii) $\lim _{r \rightarrow \infty} n(r)=1$;

(iii) $\lim _{r \rightarrow \infty} \int_{1}^{r}(1-n(t)) t^{-1} d t$ exists (and is finite).

The representation (1.8) follows. For simplicity we assume

$$
u(0)-\int \log |s| d \mu(s)=0 .
$$

LEMma 1.3. Under the hypothesis (1.9) on $u$,

$$
\limsup _{|t| \rightarrow \infty} \int \log \frac{|t-s|}{|t|} d \mu(s) \leq 0 .
$$

Proof. Fix $t \in \mathbb{C}$ with $|t|>1$ and a positive integer $k$. We split the integral into two parts:

$$
\begin{aligned}
& \text { if }|s| \leq|t| / k \text {, then } \frac{|t-s|}{|t|} \leq \frac{|t|+|s|}{|t|} \leq \frac{k+1}{k} \\
& \text { if }|s| \geq|t| / k \text {, then } \frac{|t-s|}{|t|} \leq \frac{|t|+|s|}{|t|} \leq \frac{(k+1)|s|}{|t|} .
\end{aligned}
$$


Then for the first part we have

$$
\int_{|s| \leq|t| / k} \log \frac{|t-s|}{|t|} d \mu(s) \leq \log \left(\frac{k+1}{k}\right) \cdot n(|t| / k) \leq \log \left(\frac{k+1}{k}\right) ;
$$

while for the second part,

$$
\begin{aligned}
& \int_{|s| \geq|t| / k} \log \frac{|t-s|}{|t|} d \mu(s) \\
& \leq \int_{|s| \geq|t| / k} \log [(k+1)|s|] d \mu(s)-\int_{|s| \geq|t| / k} \log |t| d \mu(s) \\
& =\log (k+1) \cdot[1-n(|t| / k)]+\int_{|s| \geq|t| / k} \log |s| d \mu(s)-\log |t| \cdot[1-n(|t| / k)] \\
& \leq \log (k+1) \cdot[1-n(|t| / k)]+\int_{|s| \geq|t| / k} \log |s| d \mu(s)
\end{aligned}
$$

Using (i) and (ii) we obtain

$$
\limsup _{|t| \rightarrow \infty} \int \log \frac{|t-s|}{|t|} d \mu(s) \leq \log \left(\frac{k+1}{k}\right)
$$

and the result follows.

We now show that by suitably averaging the function $u$, we will get existence of the limit above with $u$ replaced by this averaged version. Precisely, fix $r>0$ and define

$$
u^{r}(t):=\frac{1}{2 \pi} \int_{0}^{2 \pi} u\left(t+r e^{i \theta}\right) d \theta
$$

Then $u^{r} \in L^{+}(\mathbb{C}) \cap C(\mathbb{C})$ and $u^{r}$ satisfies (1.9) if $r<\delta$ with

$$
d \mu^{r}(t)=\frac{-1}{4 \pi i} \Delta u^{r}(t) d t \wedge d \bar{t} ;
$$

thus

$$
\limsup _{|t| \rightarrow \infty} \int \log \frac{|t-s|}{|t|} d \mu^{r}(s) \leq 0 .
$$

LEMma 1.4. $\lim _{|t| \rightarrow \infty}\left[u^{r}(t)-\log |t|\right]=0$.

Proof. It suffices to show

$$
\liminf _{|t| \rightarrow \infty}\left[u^{r}(t)-\log |t|\right] \geq 0 .
$$

Fix $t \in \mathbb{C}$ with $|t|>1$. For simplicity, take $r=1$. By (ii), (iii) and Fubini's theorem, we can write 


$$
\begin{aligned}
u^{1}(t)-\log |t| & =\int \log ^{+}|t-s| d \mu(s)-\int \log |t| d \mu(s) \\
& =\int_{|t-s| \geq 1} \log \frac{|t-s|}{|t|} d \mu(s)-\int_{|t-s| \leq 1} \log |t| d \mu(s) .
\end{aligned}
$$

The second term is equal to $n(t ; 1) \log |t|$ where $n(t ; 1):=\int_{|t-s| \leq 1} d \mu(s)$ is the mass of the measure $\mu$ in the disk of radius 1 centered at $t$; clearly

$$
n(t ; 1) \leq n(|t|+1)-n(|t|-1)
$$

so that

$$
n(t ; 1) \log |t| \leq C \int_{|t|-1}^{|t|+1} \log r d n(r)
$$

for some constant $C$ which is independent of $t$. By (i), we see that

$$
\lim _{|t| \rightarrow \infty} n(t ; 1) \log |t|=0 .
$$

Let

$$
G(t):=\int_{|t-s| \geq 1} \log \frac{|t-s|}{|t|} d \mu(s) .
$$

From (1.10), we must show $\liminf _{|t| \rightarrow \infty} G(t) \geq 0$. Clearly we need only consider the nonpositive part

$$
G^{-}(t):=\int_{1 \leq|t-s| \leq|t|} \log \frac{|t-s|}{|t|} d \mu(s)
$$

and show that $\liminf _{|t| \rightarrow \infty} G^{-}(t) \geq 0$.

To this end, fix $\varepsilon>0$ and split up $G^{-}(t)$ into two parts:

$$
\begin{aligned}
G^{-}(t):= & \int_{1 \leq|t-s| \leq|t|,|s| \leq \varepsilon|t|} \log \frac{|t-s|}{|t|} d \mu(s) \\
& +\int_{1 \leq|t-s| \leq|t|,|s| \geq \varepsilon|t|} \log \frac{|t-s|}{|t|} d \mu(s) .
\end{aligned}
$$

In the first integral, we have

$$
\frac{|t-s|}{|t|} \geq \frac{|t|-|s|}{|t|} \geq 1-\varepsilon
$$

so that $\log (|t-s|) /|t| \geq \log (1-\varepsilon)=O(\varepsilon)$. We split up the second integral in (1.11) into two parts: one with $|s| \geq|t|$ and one with $|s| \leq|t|$. Defining

$$
U(\varepsilon, t):=\{s: 1 \leq|t-s| \leq|t| \text { and } \varepsilon|t| \leq|s| \leq|t|\}
$$


for $s \in U(\varepsilon, t)$ we have

$$
\frac{|t-s|}{|t|} \geq \frac{1}{|t|} \geq \frac{\varepsilon}{|s|}
$$

Thus

$$
\begin{aligned}
\int_{U(\varepsilon, t)} \log \frac{|t-s|}{|t|} d \mu(s) & \geq \int_{U(\varepsilon, t)} \log \varepsilon d \mu(s)-\int_{U(\varepsilon, t)} \log |s| d \mu(s) \\
& \geq \log \varepsilon \cdot[n(|t|)-n(\varepsilon|t|)]-\int_{\varepsilon|t|}^{|t|} \log r d n(r) .
\end{aligned}
$$

We may assume $\varepsilon|t|>1$ since we are interested (fixing $\varepsilon>0$ ) in the behavior of $G^{-}(t)$ for $|t|$ large. For $s$ satisfying $|s| \geq|t|$ and $1 \leq|t-s| \leq|t|$,

$$
\frac{|t-s|}{|t|} \geq \frac{1}{|t|} \geq \frac{1}{|s|}
$$

Hence

$\int_{1 \leq|t-s| \leq|t|,|s| \geq|t|} \log \frac{|t-s|}{|t|} d \mu(s) \geq-\int_{|s| \geq|t|} \log |s| d \mu(s)=-\int_{r \geq|t|} \log r d n(r)$.

Altogether, we obtain the estimate

$$
\begin{aligned}
\liminf _{|t| \rightarrow \infty} G^{-}(t) \geq & \liminf _{|t| \rightarrow \infty}\{O(\varepsilon) n(\varepsilon|t|)+\log \varepsilon \cdot[n(|t|)-n(\varepsilon|t|)] \\
& \left.-\int_{\varepsilon|t|}^{|t|} \log r d n(r)-\int_{r \geq|t|} \log r d n(r)\right\} .
\end{aligned}
$$

Again using (i) and (ii), we have

$$
\liminf _{|t| \rightarrow \infty} G^{-}(t) \geq \liminf _{|t| \rightarrow \infty} O(\varepsilon) n(\varepsilon|t|)=O(\varepsilon)
$$

and the result follows.

We will use these results in Section 4 when we discuss the existence of directional limits for Robin functions $\varrho_{u}$ associated to functions $u \in L$ in $\mathbb{C}^{N}, N>1$.

2. Computing $V_{K}$ using one-variable methods. This section is essentially contained in [BCL]. It contains the primary motivation for our results; for omitted proofs we refer the reader to [BCL]. Let $K \subset \mathbb{C}^{N}$ be compact. We recall that $K$ is nonpluripolar as a subset of $\mathbb{C}^{N}$ if and only if $V_{K}^{*} \in L$ (equivalently, $V_{K}^{*} \not \equiv \infty$ ) and that $K$ is regular if and only if $V_{K}^{*}=V_{K}$ (equivalently, $V_{K}$ is continuous on $\mathbb{C}^{N}$ ). Moreover, if we let

$$
\widehat{K}:=\left\{z \in \mathbb{C}^{N}:\left|p\left(z_{1}, \ldots, z_{N}\right)\right| \leq\|p\|_{K} \text { for all polynomials } p\right\}
$$


denote the polynomial hull of $K$, then $\widehat{K}=\left\{z \in \mathbb{C}^{N}: V_{K}(z)=0\right\}$ and $V_{\widehat{K}}=V_{K}$.

We recall from [BCL] how to relate the notions of regularity and (pluri-) polarity in one and several variables for $K$ and $p(K)$ when $p$ is a nonconstant polynomial.

Lemma $2.1[\mathrm{BCL}]$. Suppose that $E \subset \mathbb{C}^{N}$ is a bounded Borel set and that $p: \mathbb{C}^{N} \rightarrow \mathbb{C}$ is a nonconstant polynomial. Then (a) if $E$ is nonpluripolar, $p(E)$ is nonpolar, and (b) if $E$ is a regular compact set, then $p(E)$ is regular.

If $K$ is compact and regular and $p_{d}$ is a polynomial of degree $d$, then

$$
\frac{1}{d} V_{p_{d}(K)}\left(p_{d}(z)\right) \leq V_{K}(z) ;
$$

conversely, if $\left\|p_{d}\right\|_{K} \leq 1$, then $p_{d}(K) \subset U$, the unit disk in $\mathbb{C}$, so that $V_{p_{d}(K)}(w) \geq V_{U}(w)=\log ^{+}(|w|)$ for all $w \in \mathbb{C}$, so $V_{p_{d}(K)}\left(p_{d}(z)\right) \geq \log ^{+}\left(\left|p_{d}(z)\right|\right)$, from which it follows that

$$
V_{K}(z) \leq \sup _{p_{d}} \frac{1}{d} V_{p_{d}(K)}\left(p_{d}(z)\right) .
$$

Thus

$$
V_{K}(z)=\sup _{p_{d}} \frac{1}{d} V_{p_{d}(K)}\left(p_{d}(z)\right) .
$$

If $d=1$, this implies that for any complex affine function $\ell(z)$, we have

$$
V_{\ell(K)}(\ell(z)) \leq V_{K}(z) \text {. }
$$

Define

$$
V^{(1)}(z):=\sup \left\{V_{\ell(K)}(\ell(z)): \ell \in\left(\mathbb{C}^{N}\right)^{*}, \ell \neq 0\right\}
$$

where $\left(\mathbb{C}^{N}\right)^{*}$ is the class of all complex-linear functionals on $\mathbb{C}^{N}$. Note that if we replace $\ell$ by a scalar multiple $t \ell$, then $V_{t \ell(K)} \circ t \ell=V_{\ell(K)} \circ \ell$. Thus considering upper envelopes over all complex-linear functionals or simply, e.g., over all linear functionals normalized to have norm 1 , yields the same function $V^{(1)}$; similarly, if $\ell \in\left(\mathbb{C}^{N}\right)^{*}$ and $a \in \mathbb{C}$ is constant, then we have $V_{(\ell+a)(K)}((\ell+a)(z))=V_{\ell(K)}(\ell(z))$. If $E \subset \mathbb{C}^{N}$ is a bounded Borel set, we define

$$
V^{(1)}(z):=\sup \left\{V_{\ell(E)}^{*}(\ell(z)): \ell \in\left(\mathbb{C}^{N}\right)^{*}, \ell \neq 0\right\}
$$

and by [K, Corollary 5.2.5] it follows that $V^{(1) *} \leq V_{E}^{*}$.

Returning to the case where $K$ is compact and regular, note that $V^{(1)}$ is lower semicontinuous as the upper envelope of a family of continuous functions. Since we will show (Proposition 3.5) that in this setting, $V^{(1)}$ is actually continuous, it is natural to ask for the most general situation under which we have the equality $V^{(1)}=V_{K}$. A necessary condition is given in $[\mathrm{BCL}]$. 
Proposition 2.2 [BCL]. Let $N>1$. Suppose $K \subset \mathbb{C}^{N}$ is compact, regular, and polynomially convex $(K=\widehat{K})$. Define $V^{(1)}(z)$ using $(2.2)$. If $V^{(1)}(z)=V_{K}(z)$ in $\mathbb{C}^{N}$, then $K$ is lineally convex; i.e., the complement of $K$ is the union of complex hyperplanes.

For each positive integer $n$, we can define

$$
V^{(n)}(z)=V_{K}^{(n)}(z):=\sup \left\{\frac{1}{\operatorname{deg} p} V_{p(K)}(p(z)): 1 \leq \operatorname{deg} p \leq n\right\} .
$$

Equation (2.1) shows that, for any regular compact set $K$, the functions $V^{(n)}$ increase monotonically to $V_{K}$; i.e.,

$$
V^{(n)} \leq V^{(n+1)}, \quad n=1,2, \ldots, \quad \text { and } \quad \lim _{n \rightarrow \infty} V^{(n)}(z)=V_{K}(z), \quad z \in \mathbb{C}^{N} .
$$

We study the functions $V^{(n)}$ in the rest of the paper.

Note that if $K$ is nonpluripolar, then $V^{*}:=V^{(1) *}$ (and hence $V^{(n) *}$ for each $n=1,2, \ldots)$ is in the class $L^{+}$where

$$
L^{+}:=\left\{u \in L: \log ^{+}|z|+C_{1} \leq u(z) \leq \log ^{+}|z|+C_{2} \text { for some } C_{1}, C_{2}\right\} .
$$

For it is well known that $V_{K}^{*} \in L^{+}$if $K$ is nonpluripolar; letting $\ell_{j}(z)=z_{j}$, $j=1, \ldots, N$, we have

$$
V_{K}^{*}(z) \geq V_{K}(z) \geq V^{(1)}(z) \geq \max _{j=1, \ldots, N} V_{\ell_{j}(K)}\left(\ell_{j}(z)\right) .
$$

But $\max _{j=1, \ldots, N} V_{\ell_{j}(K)}\left(\ell_{j}(z)\right)=V_{\ell_{1}(K) \times \ldots \times \ell_{N}(K)}(z)$ and $V_{\ell_{1}(K) \times \ldots \times \ell_{N}(K)}^{*}$ $\in L^{+}$since each $\ell_{j}(K)$ is nonpolar by Lemma 2.1.

Finally, we note that if $N=1$, then $V^{(1)}=V_{K}$ for all compact sets $K$.

3. Continuity of $V^{(n)}$. In this section, we will always assume that $K \subset \mathbb{C}^{N}$ is compact and regular; moreover, we may assume $K \subset B$, the unit ball. Our main task in this section is to show that each of the functions $V^{(n)}=V_{K}^{(n)}, n=1,2, \ldots$, is continuous. We first work with $V^{(1)}$ and see which results generalize. Recall that we may assume our linear functionals $\ell$ are normalized to have norm 1 ; in the case of $V^{(n)}$ for $n>1$, since $V_{t p(K)} \circ$ $t p=V_{p(K)} \circ p$, we are again free to normalize in an appropriate fashion. For example, writing $p=H_{n}+H_{n-1}+\ldots+H_{0}$ where $H_{k}$ is a homogeneous polynomial of degree $k$, we may require that $\left\|H_{n}\right\|_{B}=1$. We begin by stating a lemma which will be useful in the next section in proving continuity of $\varrho_{V^{(1)}}$.

Lemma 3.0. Fix a positive integer $n$. If $K \subset \mathbb{C}^{N}$ is compact and regular, then

$$
\inf _{p} \operatorname{cap}(p(K))>0
$$

where the infimum is taken over all nonconstant polynomials $p=H_{n}+$ $H_{n-1}+\ldots+H_{0}$ of degree at most $n$ with $\left\|H_{n}\right\|_{B}=1$. 
Proof. We know from the previous section that $V^{(n)} \in L^{+}$; in particular, there exists a constant $C$ so that for $|z|>1$, we have $V^{(n)}(z) \leq C+\log |z|$. Thus for any $p$,

$$
\frac{1}{n} V_{p(K)}(p(z)) \leq C+\log |z|, \quad|z|>1 .
$$

For motivational purposes, we first give a proof for the case $n=1$ (linear case) using this normalization: for a linear functional $\ell(z)=a_{1} z_{1}+$ $\ldots+a_{N} z_{N}$, we suppose $\left|a_{1}\right|^{2}+\ldots+\left|a_{N}\right|^{2}=1$. Given $t \in \mathbb{C}$, setting $z_{1}=t \bar{a}_{1}, \ldots, z_{N}=t \bar{a}_{N}$ yields a point $z \in \mathbb{C}^{N}$ with $\ell(z)=t$ and $|z|=|t|$. Thus for such $z$ and $t$ with $|z|=|t|>1$, we have

$$
V_{\ell(K)}(t)=V_{\ell(K)}(\ell(z)) \leq C+\log |t|+\log \frac{|z|}{|t|}=C+\log |t| .
$$

Letting $|t| \rightarrow \infty$, we conclude that $\varrho_{\ell(K)} \leq C$ so that $\operatorname{cap}(\ell(K))>e^{-C}$.

For the general case, write $p(z):=t, t \in \mathbb{C}$. Then if $|z|>1$ and $t \neq 0$,

$$
V_{p(K)}(t)=V_{p(K)}(p(z)) \leq n C+\log |t|+n \log \frac{|z|}{|t|^{1 / n}} .
$$

Now since $\left\|H_{n}\right\|_{B}=1$, for any $R \geq 1,\left\|H_{n}\right\|_{B(R)}=R^{n}$ and hence

$$
\|p\|_{B(R)} \geq R^{n}, \quad R \geq 1 .
$$

Choose a sequence $\left\{R_{k}\right\}$ of radii each larger than $R_{0}$ and increasing to $\infty$, and choose corresponding points $\left\{z_{k}\right\}$ with $\left|z_{k}\right|=R_{k}$ such that $\left|p\left(z_{k}\right)\right|=$ : $\left|t_{k}\right|=\|p\|_{B\left(R_{k}\right)} \geq R_{k}^{n}$. Then $\left|t_{k}\right| \uparrow \infty$ and, since

$$
\frac{\left|z_{k}\right|}{\left|t_{k}\right|^{1 / n}} \leq \frac{R_{k}}{R_{k}}=1
$$

for the points $t_{k}$ we have

$$
V_{p(K)}\left(t_{k}\right) \leq n C+\log \left|t_{k}\right| .
$$

Letting $k \rightarrow \infty$, we have $\varrho_{p(K)} \leq n C$ so that $\operatorname{cap}(p(K))>e^{-n C}$. Note we are using the fact that for planar (nonpolar) compact sets, such as $E=p(K)$, the limit

$$
\lim _{t \rightarrow \infty}\left[V_{E}(t)-\log |t|\right]=\varrho_{E}
$$

exists.

In the next few results, we use the fact that for regular compact sets $E, F$ in $\mathbb{C}^{N}$ (even $\left.N=1\right)$,

$$
\left\|V_{E}-V_{F}\right\|_{\mathbb{C}^{N}}=\left\|V_{E}-V_{F}\right\|_{E \cup F}=\max \left[\left\|V_{E}\right\|_{F},\left\|V_{F}\right\|_{E}\right] .
$$

For $K \subset \mathbb{C}^{N}$ and $\delta>0$, we define

$$
K^{\delta}:=\left\{z \in \mathbb{C}^{N}: \operatorname{dist}(z, K) \leq \delta\right\} .
$$


Lemma 3.1. Let $K \subset \mathbb{C}^{N}$ be compact and regular. Given $\varepsilon>0$, there exists $\delta>0$ such that if $K^{\prime}$ is compact and regular and

$$
K \subset\left(K^{\prime}\right)^{\delta}, \quad K^{\prime} \subset K^{\delta},
$$

then $\left\|V_{K}-V_{K^{\prime}}\right\|_{\mathbb{C}^{N}} \leq \varepsilon$.

Proof. Since $K^{\delta},\left(K^{\prime}\right)^{\delta}$ decrease to $K, K^{\prime}$ as $\delta$ decreases to 0 , we can choose $\delta$ so that

$$
K^{\delta} \subset\left\{z \in \mathbb{C}^{N}: V_{K}(z)<\varepsilon\right\} \quad \text { and } \quad\left(K^{\prime}\right)^{\delta} \subset\left\{z \in \mathbb{C}^{N}: V_{K^{\prime}}(z)<\varepsilon\right\} .
$$

Then for all $z \in \mathbb{C}^{N}$,

$$
V_{K}(z)-\varepsilon \leq V_{K^{\delta}}(z) \quad \text { and } \quad V_{K^{\prime}}(z)-\varepsilon \leq V_{\left(K^{\prime}\right)^{\delta}}(z) .
$$

By (3.2), $V_{\left(K^{\prime}\right)^{\delta}} \leq V_{K}$ and $V_{K^{\delta}} \leq V_{K^{\prime}}$; combining with the above equation, we obtain

$$
V_{K}(z)-\varepsilon \leq V_{K^{\delta}}(z) \leq V_{K^{\prime}}(z) \quad \text { and } \quad V_{K^{\prime}}(z)-\varepsilon \leq V_{\left(K^{\prime}\right)^{\delta}}(z) \leq V_{K}(z) ;
$$

i.e., $\left\|V_{K}-V_{K^{\prime}}\right\|_{\mathbb{C}^{N}} \leq \varepsilon$.

Corollary 3.2. Given $\varepsilon>0$, there exists $\delta>0$ such that if $T: \mathbb{C}^{N} \rightarrow$ $\mathbb{C}^{N}$ is an invertible linear transformation with $\|T-I\|,\left\|T^{-1}-I\right\|<\delta$, then

$$
\left\|V_{K}-V_{T(K)}\right\|_{\mathbb{C}^{N}}<\varepsilon
$$

Proof. We know that given $\varepsilon>0$, there exists $\delta>0$ such that if $K^{\prime}$ is compact and regular and

$$
K \subset\left(K^{\prime}\right)^{\delta}, \quad K^{\prime} \subset K^{\delta},
$$

then $\left\|V_{K}-V_{K^{\prime}}\right\|_{\mathbb{C}^{N}} \leq \varepsilon$. If $\|T-I\|,\left\|T^{-1}-I\right\|<\delta$, since $K \subset B$, for $z \in T(K)$ we have

$$
\operatorname{dist}(z, K) \leq\left|T^{-1}(z)-z\right|<\delta
$$

This says that $T(K) \subset K^{\delta}$. Similarly, for $z \in K$ we have

$$
\operatorname{dist}(z, T(K)) \leq|T(z)-z|<\delta .
$$

This says that $K \subset(T(K))^{\delta}$ and the result follows.

Lemma 3.3. Let $T: \mathbb{C}^{N} \rightarrow \mathbb{C}^{N}$ be an invertible linear transformation. For any $\mathbb{C}$-linear $\ell: \mathbb{C}^{N} \rightarrow \mathbb{C}$ with $\ell \not \equiv 0$,

$$
\left\|V_{\ell(K)}-V_{\ell(T(K))}\right\|_{\mathbb{C}} \leq\left\|V_{K}-V_{T(K)}\right\|_{\mathbb{C}^{N}} .
$$

Proof. From (3.1), we need only estimate $\left|V_{\ell(K)}(w)-V_{\ell(T(K))}(w)\right|$ at points $w \in \ell(K) \cup \ell(T(K))$. Fix $w \in \ell(T(K))$. Then $V_{\ell(T(K))}(w)=0$, and, writing $w=\ell(T(z))$ for some $z \in K$, we have

$$
\begin{aligned}
V_{\ell(K)}(w) & =V_{\ell(K)}(\ell(T(z)))=\left[V_{\ell(K)} \circ \ell\right](T(z)) \\
& \leq V_{K}^{(1)}(T(z)) \leq V_{K}(T(z)) \leq\left\|V_{K}\right\|_{T(K)} .
\end{aligned}
$$


Similarly, if $w \in \ell(K)$, we obtain the inequality $V_{\ell(T(K))}(w) \leq\left\|V_{T(K)}\right\|_{K}$. The result follows from (3.1).

We will need the following linear algebra lemma.

Lemma 3.4. Fix $z \in \mathbb{C}^{N} \backslash\{0\}$ and $0<\delta<1 / 2$. For each $z^{\prime} \in$ $B(z, \delta|z|):=\left\{z^{\prime}:\left|z-z^{\prime}\right|<\delta|z|\right\}$, there exists an invertible linear transformation $T: \mathbb{C}^{N} \rightarrow \mathbb{C}^{N}$ with $T(z)=z^{\prime}$ and $\|T-I\|,\left\|T^{-1}-I\right\|<2 \delta$.

Proof. For simplicity, we take $z=\left(z_{1}, 0, \ldots, 0\right)$. Define $T$ on the standard basis vectors $e_{j}:=(0, \ldots, 0,1,0, \ldots, 0)$ ( 1 in the $j$ th slot) by

$$
T\left(e_{1}\right)=T(z) / z_{1}:=z^{\prime} / z_{1}, \quad T\left(e_{j}\right)=e_{j}, \quad j=2, \ldots, n .
$$

For a vector $w=\left(w_{1}, \ldots, w_{N}\right) \in \mathbb{C}^{N}$, we have $|w-T(w)|=\left|1-z_{1}^{\prime} / z_{1}\right|\left|w_{1}\right|$ so that

$$
\|T-I\| \leq\left|1-z_{1}^{\prime} / z_{1}\right|=\left|\frac{z_{1}-z_{1}^{\prime}}{z_{1}}\right| \leq\left|z-z^{\prime}\right| /|z|<\delta|z| /|z|=\delta .
$$

Since $\left\|T^{-1}-I\right\| \leq \delta /(1-\delta)<2 \delta$, the result follows.

Proposition 3.5. For $K$ regular, $V_{K}^{(1)}$ is continuous on $\mathbb{C}^{N}$.

Proof. Given $\varepsilon>0$, choose $\delta>0$ as in Corollary 3.2. Then for $T$ : $\mathbb{C}^{N} \rightarrow \mathbb{C}^{N}$ an invertible linear transformation with $\|T-I\|,\left\|T^{-1}-I\right\|<\delta$, we obtain

$$
\left\|V_{K}-V_{T(K)}\right\|_{\mathbb{C}^{N}}<\varepsilon
$$

For such a $T$, by Lemma 3.3, if $\ell: \mathbb{C}^{N} \rightarrow \mathbb{C}$ is linear with $\ell \not \equiv 0$,

$$
\left\|V_{\ell(K)}-V_{\ell(T(K))}\right\|_{\mathbb{C}} \leq\left\|V_{K}-V_{T(K)}\right\|_{\mathbb{C}^{N}}<\varepsilon .
$$

We claim that (3.3) implies that

$$
\left\|V_{K}^{(1)}-V_{T(K)}^{(1)}\right\|_{\mathbb{C}^{N}}<\varepsilon .
$$

For, given any $z \in \mathbb{C}^{N},(3.3)$ gives

$$
\left|V_{\ell(K)}(\ell(z))-V_{\ell(T(K))}(\ell(z))\right|<\varepsilon .
$$

Thus

$$
V_{\ell(K)}(\ell(z)) \leq \varepsilon+V_{\ell(T(K))}(\ell(z)) \leq \varepsilon+V_{T(K)}^{(1)}(z) .
$$

As this holds for all $\ell: \mathbb{C}^{N} \rightarrow \mathbb{C}$ with $\ell \neq \equiv 0$,

$$
V_{K}^{(1)}(z) \leq \varepsilon+V_{T(K)}^{(1)}(z) .
$$

Reversing the roles of $K$ and $T(K)$ together with the above inequality yields (3.4). 
However,

$$
\begin{aligned}
V_{T(K)}^{(1)}(z) & =\sup \left\{V_{\ell(T(K))}(\ell(z)): \ell \not \equiv 0\right\} \\
& =\sup \left\{V_{\ell(T(K))}\left(\ell \circ T\left(T^{-1}(z)\right)\right): \ell \not \equiv 0\right\}=V_{K}^{(1)}\left(T^{-1}(z)\right) .
\end{aligned}
$$

Combining with (3.4) gives

$$
\left|V_{K}^{(1)}(z)-V_{K}^{(1)}\left(T^{-1}(z)\right)\right|<\varepsilon
$$

for all $z \in \mathbb{C}^{N}$. Fixing $z \in \mathbb{C}^{N} \backslash\{0\}$, and setting $\delta^{\prime}=\delta^{\prime}(z, \varepsilon):=\delta|z| / 2$, by Lemma 3.4, for each $z^{\prime}$ with $\left|z-z^{\prime}\right|<\delta^{\prime}=\delta|z| / 2$, we can find $T$ as above with $T^{-1}(z)=z^{\prime}$. Thus, applying (3.5), we have shown that $\left|z-z^{\prime}\right|<\delta^{\prime}$ implies that $\left|V_{K}^{(1)}(z)-V_{K}^{(1)}\left(z^{\prime}\right)\right|<\varepsilon$; i.e., $V_{K}^{(1)}$ is continuous at $z$. For $z=0$, we observe that for any $a \in \mathbb{C}^{N}, V_{K}^{(1)}(z)=V_{K+a}^{(1)}(z+a)$; hence continuity of $V_{K}^{(1)}$ at 0 follows from continuity of $V_{K+a}^{(1)}$ at $a$.

Note that this argument generalizes to show that $V_{K}^{(n)}$ is continuous for $n=1,2, \ldots$ Lemmas 3.1, 3.4 and Corollary 3.2 are general facts about linear transformations and (usual) extremal functions. Lemma 3.3 remains valid upon replacing $\ell$ by a nonconstant polynomial $p_{d}$; hence the argument of Proposition 3.5 can be repeated virtually line-by-line to obtain continuity of $V_{K}^{(n)}$ (note that $T, T^{-1}$ invertible, $p_{d}$ a nonconstant polynomial implies $p_{d} \circ T, p_{d} \circ T^{-1}$ are nonconstant polynomials of the same degree as $\left.p_{d}\right)$. Thus we may state the following.

Proposition $3.5^{\prime}$. For $K$ regular, $V_{K}^{(n)}$ is continuous on $\mathbb{C}^{N}, n=$ $1,2, \ldots$

4. Existence of directional limits and continuity of $\varrho_{V^{(1)}}$. We begin with a general fact about extremal functions in one variable. Let $\Delta$ denote the unit disk in $\mathbb{C}$.

Lemma 4.1. Let $X \subset \Delta$ be nonpolar. For $|\eta| \leq 1,|\xi| \gg 1$,

$$
\left|V_{X}^{*}(\xi+\eta)-V_{X}^{*}(\xi)\right|=|\eta| /|\xi|+O\left(|\eta| /|\xi|^{2}\right)
$$

where $O\left(|\eta| /|\xi|^{2}\right)$ is independent of $X$.

Proof. Consider

$$
\begin{aligned}
\left|V_{X}^{*}(\xi+\eta)-V_{X}^{*}(\xi)\right| & =\left|\int_{X} \log \frac{|\xi+\eta-t|}{|\xi-t|} d \mu_{X}(t)\right| \\
& =\left|\log \frac{|\xi+\eta|}{|\xi|}+\int_{X} \log \frac{|\xi+\eta-t||\xi|}{|\xi-t||\xi+\eta|} d \mu_{X}(t)\right| \\
& =:|\log | 1+\frac{|\eta|}{|\xi|}|+R(\xi, \eta)|
\end{aligned}
$$


where

$$
R(\xi, \eta):=\int_{X} \log \frac{|\xi+\eta-t||\xi|}{|\xi-t||\xi+\eta|} d \mu_{X}(t)
$$

Now

$$
\begin{aligned}
\frac{|\xi+\eta-t||\xi|}{|\xi-t||\xi+\eta|} & =\frac{\left|\xi^{2}+\xi(\eta-t)\right|}{\left|\xi^{2}+\xi(\eta-t)-t \eta\right|} \\
& \leq \frac{\left|\xi^{2}+\xi(\eta-t)\right|}{\left|\xi^{2}+\xi(\eta-t)\right|-|t \eta|}=\frac{1}{1-|t \eta| /\left|\xi^{2}+\xi(\eta-t)\right|} \\
& =1+O\left(|\eta| /|\xi|^{2}\right)
\end{aligned}
$$

(note that $|\eta|,|t| \leq 1$ ). Similarly,

$$
\frac{|\xi+\eta-t||\xi|}{|\xi-t||\xi+\eta|} \geq \frac{1}{1+|t \eta| /\left|\xi^{2}+\xi(\eta-t)\right|}=1-O\left(|\eta| /|\xi|^{2}\right) .
$$

Thus, since $\mu_{X}(X)=1$,

$$
|R(\xi, \eta)|=\log \left(1+O\left(|\eta| /|\xi|^{2}\right)\right)=O\left(|\eta| /|\xi|^{2}\right) .
$$

Finally,

$$
|\log | 1+\frac{|\eta|}{|\xi|}|| \leq \frac{|\eta|}{|\xi|}+O\left(|\eta|^{2} /|\xi|^{2}\right) \leq \frac{|\eta|}{|\xi|}+O\left(|\eta| /|\xi|^{2}\right)
$$

and the result follows. We only use $\mu_{X}(X)=1$ and $X$ nonpolar so $O\left(|\eta| /|\xi|^{2}\right)$ is independent of $X$.

We write $V:=V_{K}^{(1)}$ below for simplicity.

Corollary 4.2. Let $K \subset B \subset \mathbb{C}^{N}$ be a regular compact set. There exists $M \geq 1$ such that given any $\varepsilon>0$, there exists $R=R(K, \varepsilon)>1$ with

$$
|V(z+\eta)-V(z)| \leq M|\eta| /|z|+\varepsilon \quad \text { for all }|\eta| \leq 1,|z|>R .
$$

Proof. Note that for $\ell$ normalized so that $\|\ell\|=1$, each set $\ell(K)$ is a compact, nonpolar subset of $\Delta$. Thus, by the lemma,

$$
\left|V_{\ell(K)}(t+s)-V_{\ell(K)}(t)\right| \leq|s| /|t|+O\left(|s| /|t|^{2}\right), \quad|s| \leq 1,|t| \gg 1 .
$$

Since $O\left(|s| /|t|^{2}\right)$ is independent of $\ell$, we can choose $R^{\prime} \gg 1$ so that

$$
\left|V_{\ell(K)}(t+s)-V_{\ell(K)}(t)\right| \leq 2|s| /|t|, \quad|s| \leq 1,|t|>R^{\prime},
$$

for all $\ell$.

Since $K$ is nonpluripolar, $V \in L^{+}$(cf. Section 2); thus, there exist $C_{1}, C_{2}$ with

$$
\log ^{+}|z|+C_{1} \leq V(z) \leq \log ^{+}|z|+C_{2}
$$


in all of $\mathbb{C}^{N}$. By Lemma 3.0, $\operatorname{cap}(\ell(K)) \geq a$ for some $a>0$ if $\|\ell\|=1$. Thus, there exists $c^{\prime}=c^{\prime}(a)>0$ such that

$$
\log ^{+}|w| \leq V_{\ell(K)}(w) \leq \log ^{+}|w|+c^{\prime}
$$

for all $\|\ell\|=1$ and all $w \in \mathbb{C}$.

Next, choose $R>R^{\prime}$ so that $\log R \gg \max \left[\left|C_{1}\right|,\left|C_{2}\right|, \varepsilon, c^{\prime}\right]$. Given $|z|>R$, choose $\ell=\ell_{z}$ such that

$$
V(z) \geq V_{\ell(K)}(\ell(z)) \geq V(z)-\varepsilon .
$$

Combining (4.1), (4.2) and (4.3) for $\ell=\ell_{z}$ at the point $z$ we obtain

$$
\log ^{+}|\ell(z)|+\left(c^{\prime}-C_{1}\right) \geq \log ^{+}|z|-\varepsilon
$$

By the choice of $R$, we have

$$
|\ell(z)| \geq \frac{|z|}{e^{\varepsilon+c^{\prime}-C_{1}}} \geq b_{1}|z|
$$

where $b_{1}=b_{1}(a):=1 / e^{1+c^{\prime}-C_{1}}$ (we may assume $\varepsilon<1$ ). Note that as long as $\varepsilon<1$, the constant $b_{1}$ depends only on $K$ (from (4.1)) and hence $a$ (from (4.2)). Thus we are free to take $R=R(K, \varepsilon)$ sufficiently large so that, e.g., $R>4 / b_{1}$. This we do.

Now given $|\eta| \leq 1,|z|>R$, choose $\ell=\ell_{z}$ so that (4.3) holds and $\ell_{\eta}$ so that

$$
V(z+\eta) \geq V_{\ell_{\eta}(K)}\left(\ell_{\eta}(z+\eta)\right) \geq V(z+\eta)-\varepsilon
$$

and $\left|\ell_{\eta}(z+\eta)\right| \geq b_{1}|z+\eta|$ so that

$$
\left|\ell_{\eta}(z)\right| \geq b_{1}|z|-b_{1}|\eta|-1 \geq b_{1}|z|-\left(b_{1}+1\right) \geq b|z|
$$

where $b=b\left(b_{1}\right)$ (since we may assume $R>4 / b_{1}$ (so $|z|>4 / b_{1}$ ) and take $\left.b=b_{1} / 2\right)$. Using (4.3), (4.4) and the fact that

$$
V_{\ell_{\eta}(K)}\left(\ell_{\eta}(z)\right) \leq V(z), \quad V_{\ell(K)}(\ell(z+\eta)) \leq V(z+\eta),
$$

we obtain

$$
\begin{aligned}
|V(z+\eta)-V(z)| & \leq \max \left[\frac{|\ell(\eta)|}{|\ell(z)|}+O\left(\frac{|\ell(\eta)|}{|\ell(z)|^{2}}\right), \frac{\left|\ell_{\eta}(\eta)\right|}{\left|\ell_{\eta}(z)\right|}+O\left(\frac{\left|\ell_{\eta}(\eta)\right|}{\left|\ell_{\eta}(z)\right|^{2}}\right)\right]+\varepsilon \\
& \leq \frac{1}{b}\left[\frac{|\eta|}{|z|}+O\left(\frac{|\eta|}{|z|^{2}}\right)\right]+\varepsilon
\end{aligned}
$$

where we have used the facts that $|\ell(z)|,\left|\ell_{\eta}(z)\right| \geq b|z|$ and $|\ell(\eta)|,\left|\ell_{\eta}(\eta)\right| \leq$ $|\eta|$. Since $O\left(|\eta| /|z|^{2}\right) \leq O(|\eta| /|z|)$ and this quantity is independent of $\ell, \ell_{\eta}$, the result follows.

REMARK. Note that the constant $M$ in the corollary is independent of $\varepsilon$. 
Proposition 4.3. Let $K \subset B$ be regular. For each $\alpha \in \partial B$, the directional limit

$$
\lim _{|\lambda| \rightarrow \infty}[V(\lambda \alpha)-\log |\lambda|]=\varrho_{V}(\alpha)
$$

exists.

Before proving the proposition, we recall what information we already know from the results in Section 1. Given a function $u \in L^{+}(\mathbb{C})$ (one variable), we assume that

$$
u(t):=\int \log |t-s| d \mu(s)
$$

i.e., using (1.8) and (1.9), we assume $u(0)=\int \log |s| d \mu(s)$, where

$$
d \mu(t)=\frac{-1}{4 \pi i} \Delta u(t) d t \wedge d \bar{t} .
$$

Then we showed in Section 1 that

(1) $\lim \sup _{|z| \rightarrow \infty}[u(z)-\log |z|] \leq 0$, and

(2) for $r>0, \lim _{|z| \rightarrow \infty}\left[u^{r}(z)-\log |z|\right]=0$ where

$$
u^{r}(z):=\frac{1}{2 \pi} \int_{0}^{2 \pi} u\left(z+r e^{i t}\right) d t .
$$

Thus, to verify for a given $u \in L^{+}(\mathbb{C})$ that the $\operatorname{limit} \lim _{|z| \rightarrow \infty}[u(z)-\log |z|]$ exists (recall that by Proposition 1.2, this is NOT always the case, even if $u$ is continuous), it suffices to verify that

$$
\lim _{|z| \rightarrow \infty}\left[u^{1}(z)-u(z)\right]=0 .
$$

Moreover, since $u$ is subharmonic, $u^{1}(z) \geq u(z)$ for all $z$ so that

$$
\liminf _{|z| \rightarrow \infty}\left[u^{1}(z)-u(z)\right] \geq 0 .
$$

Thus we must show:

$$
\limsup _{|z| \rightarrow \infty}\left[u^{1}(z)-u(z)\right] \leq 0 .
$$

Proof of Proposition 4.3. Fix $\alpha \in \partial B$ and consider $u(\lambda):=V(\lambda \alpha)$. Given $\varepsilon>0$, from the corollary we have

$$
u^{1}(\lambda)-u(\lambda)=\frac{1}{2 \pi} \int_{0}^{2 \pi}\left[u\left(\lambda+e^{i t}\right)-u(\lambda)\right] d t \leq \frac{M}{|\lambda|}+\varepsilon
$$

for $|\lambda|>R=R(K, \varepsilon)$. Letting $|\lambda| \rightarrow \infty$, we obtain $(*)$; i.e.,

$$
\limsup _{|\lambda| \rightarrow \infty}\left[u^{1}(\lambda)-u(\lambda)\right] \leq \varepsilon
$$

valid for all $\varepsilon>0$. 
Corollary 4.4. Let $K \subset B$ be regular. Then $\varrho_{V}$ is continuous. Moreover, we have uniformity in the limits defining the Robin function: given $\varepsilon>0$, there exists $R$ depending only on $\varepsilon$ such that for all $\alpha \in \partial B$,

$$
\varrho_{V}(\alpha)-[V(\lambda \alpha)-\log |\lambda|]<\varepsilon
$$

for $|\lambda|>R$.

Proof. We first prove the continuity. Fix $\alpha \in \partial B$. Given $\varepsilon>0$, choose $\delta>0$ as in Corollary 3.2; then for $T: \mathbb{C}^{N} \rightarrow \mathbb{C}^{N}$ an invertible linear transformation with $\|T-I\|,\left\|T^{-1}-I\right\|<\delta$, we obtain

$$
\left|V(z)-V\left(T^{-1}(z)\right)\right|<\varepsilon .
$$

In particular, for $\alpha^{\prime} \in \partial B$ with $\left|\alpha-\alpha^{\prime}\right|<\delta$, choose $T$ unitary with $T\left(\alpha^{\prime}\right)=\alpha$ and $\|T-I\|,\left\|T^{-1}-I\right\|=\left|\alpha-\alpha^{\prime}\right|<\delta$. Then if we choose $R=R\left(\varepsilon, \alpha, \alpha^{\prime}\right)$ so that for all $|\lambda|>R$,

$$
|V(\lambda \alpha)-\log | \lambda\left|-\varrho_{V}(\alpha)\right|<\varepsilon \quad \text { and } \quad\left|V\left(\lambda \alpha^{\prime}\right)-\log \right| \lambda\left|-\varrho_{V}\left(\alpha^{\prime}\right)\right|<\varepsilon,
$$

then

$$
\left|\varrho_{V}(\alpha)-\varrho_{V}\left(\alpha^{\prime}\right)\right| \leq\left|V(\lambda \alpha)-V\left(\lambda \alpha^{\prime}\right)\right|+2 \varepsilon .
$$

Since $T\left(\lambda \alpha^{\prime}\right)=\lambda \alpha$ and $\|T-I\|,\left\|T^{-1}-I\right\|<\delta$, from (3.5) we obtain

$$
\left|V(\lambda \alpha)-V\left(\lambda \alpha^{\prime}\right)\right|<\varepsilon .
$$

Thus, given $\varepsilon>0$, choosing $\delta>0$ as in Corollary 3.2 gives

$$
\left|\varrho_{V}(\alpha)-\varrho_{V}\left(\alpha^{\prime}\right)\right|<3 \varepsilon
$$

provided $\left|\alpha-\alpha^{\prime}\right|<\delta$.

For the uniformity in the limits defining the Robin function, we first note that $\varrho_{V}$ is uniformly continuous on $\partial B$; hence, given $\varepsilon>0$, there exists $\delta>0$ such that $\alpha^{\prime}, \alpha^{\prime \prime} \in \partial B$ with $\left|\alpha^{\prime}-\alpha^{\prime \prime}\right|<\delta$ implies $\left|\varrho_{V}\left(\alpha^{\prime}\right)-\varrho_{V}\left(\alpha^{\prime \prime}\right)\right|<\varepsilon$. By compactness of $\partial B$, we can choose finitely many points $\alpha_{1}, \ldots, \alpha_{m} \in \partial B$ with

$$
\partial B \subset \bigcup_{i=1}^{m}\left\{\alpha \in \partial B:\left|\alpha-\alpha_{i}\right|<\delta\right\} .
$$

Then

$$
\begin{aligned}
\varrho_{V}\left(\alpha_{i}\right) & =\liminf _{|\lambda| \rightarrow \infty}\left[V\left(\lambda \alpha_{i}\right)-\log |\lambda|\right] \\
& =\lim _{R \rightarrow \infty}\left[\inf _{|\lambda|>R}\left\{V\left(\lambda \alpha_{i}\right)-\log |\lambda|\right\}\right], \quad i=1, \ldots, m,
\end{aligned}
$$

so that there exist $R_{i}, i=1, \ldots, m$, such that

$$
V\left(\lambda \alpha_{i}\right)-\log |\lambda|>\varrho_{V}\left(\alpha_{i}\right)-\varepsilon, \quad|\lambda|>R_{i} .
$$

Set $R:=\max \left[R_{1}, \ldots, R_{m}\right]$. 
Now fix $\alpha \in \partial B$ and choose $i \in\{1, \ldots, m\}$ with $\left|\alpha-\alpha_{i}\right|<\delta$. Choose a unitary map $T$ with $T(\alpha)=\alpha_{i}$ and $\|T-I\|,\left\|T^{-1}-I\right\|<\delta$. Again, as in the proof of Proposition 3.5, we obtain

$$
\left|V(z)-V\left(T^{-1}(z)\right)\right|<\varepsilon
$$

for all $z \in \mathbb{C}^{N}$. In particular, since $T(\lambda \alpha)=\lambda \alpha_{i}$, we have

$$
\left|V(\lambda \alpha)-V\left(\lambda \alpha_{i}\right)\right|<\varepsilon
$$

for all $\lambda \in \mathbb{C}$. Then

$$
\begin{aligned}
\left\{\varrho_{V}(\alpha)-[V(\lambda \alpha)-\log |\lambda|]\right\} & -\left\{\varrho_{V}\left(\alpha_{i}\right)-\left[V\left(\lambda \alpha_{i}\right)-\log |\lambda|\right]\right\} \\
& =\varrho_{V}(\alpha)-\varrho_{V}\left(\alpha_{i}\right)+V\left(\lambda \alpha_{i}\right)-V(\lambda \alpha)<2 \varepsilon,
\end{aligned}
$$

which gives $\varrho_{V}(\alpha)-[V(\lambda \alpha)-\log |\lambda|]<3 \varepsilon$ for $|\lambda|>R$.

For completeness, we give a proof of the analogous (known) result for the Robin function $\varrho_{K}:=\varrho_{V_{K}}$ associated to the extremal function $V_{K}$ of a regular compact set. We begin with a lemma in the spirit of Corollary 4.2. We assume $K \subset B$.

Lemma 4.5. Let $z_{0} \in \mathbb{C}^{N}$ with $\left|z_{0}\right|>1$. For any $\eta \in \mathbb{C}^{N}$ with $|\eta| \leq 1$,

$$
\left|V_{K}\left(z_{0}+\eta\right)-V_{K}\left(z_{0}\right)\right| \leq \omega\left(|\eta| /\left|z_{0}\right|\right)
$$

where $\omega=\omega(\delta)$ is the modulus of continuity of $V_{K}$ on $\{z:|z| \leq 2\}$.

Proof. First of all, following the idea in the proof of Lemma 3.4, we can find an invertible linear transformation $T$ with $T\left(z_{0}\right)=z_{0}+\eta$ and $\|T-I\| \leq|\eta| /\left|z_{0}\right|$. We may assume that $V_{K}\left(z_{0}+\eta\right) \geq V_{K}\left(z_{0}\right)$. Define

$$
v(z):=\left(V_{K} \circ T\right)(z)-\omega\left(|\eta| /\left|z_{0}\right|\right) .
$$

Then $v \in L$ and if $|z| \leq 1$, we have $|T(z)| \leq 2$ so that

$$
\left|\left(V_{K} \circ T\right)(z)-V_{K}(z)\right| \leq \omega\left(|\eta| /\left|z_{0}\right|\right) \quad \text { for }|z| \leq 1 .
$$

Since $K \subset B$, this implies $v(z) \leq V_{K}(z)$ for all $z \in \mathbb{C}^{N}$ and setting $z=z_{0}$ gives the result.

Corollary 4.6. Let $K \subset \mathbb{C}^{N}$ be regular. For each $\alpha \in \partial B$, the directional limit

$$
\lim _{|\lambda| \rightarrow \infty}\left[V_{K}(\lambda \alpha)-\log |\lambda|\right]=\varrho_{K}(\alpha)
$$

exists. In addition, $\varrho_{K}$ is continuous on $\mathbb{C}^{N}$.

Proof. The existence of the directional limit follows as in the proof of Proposition 4.3 with Lemma 4.5 in place of Corollary 4.2. The continuity of $\varrho_{K}$ is then shown as in the proof of Corollary 4.4 with (3.5) replaced by $\left\|V_{K}-V_{T(K)}\right\|_{\mathbb{C}^{N}}<\varepsilon$. 
Remark. Corollary 4.6 also follows from [S1] by using the formula $V_{K}(z)=\widetilde{V}_{K}(1, z)($ see $[\mathrm{S} 1])$.

The previous result generalizes to the case of a weighted extremal function and a locally $L$-regular set $K$. Let $K \subset \mathbb{C}^{N}$ be a compact set and let $w$ be an admissible weight function on $K$; i.e., $w$ is usc and $\{z \in K: w(z)>0\}$ is not pluripolar. Let $Q:=-\log w$ and define the weighted extremal function

$$
V_{K, Q}(z):=\sup \{u(z): u \in L, u \leq Q \text { on } K\} .
$$

Next, a set $E \subset \mathbb{C}^{N}$ is said to be locally L-regular at a point $a \in \bar{E}$ if for each $r>0, V_{E \cap \bar{B}(a, r)}$ is continuous at $a$ where

$$
\bar{B}(a, r)=\left\{z \in \mathbb{C}^{N}:|z-a| \leq r\right\} .
$$

The set $E$ is locally $L$-regular if it is locally $L$-regular at each point $a \in \bar{E}$ (cf. [S2]). Clearly if $E$ is a locally $L$-regular compact set then $E$ is regular.

COROLLARY $4.6^{\prime}$. Let $K$ be a locally $L$-regular compact set and let $w \geq 0$ be a continuous weight function on $K$. Then $V_{K, Q}$ and $\varrho_{K, Q}=\varrho_{V_{K, Q}}$ are continuous. Moreover, for each $\alpha \in \partial B$, the directional limit

exists.

$$
\lim _{|\lambda| \rightarrow \infty}\left[V_{K, Q}(\lambda \alpha)-\log |\lambda|\right]=\varrho_{K, Q}(\alpha)
$$

Proof. The continuity of $V_{K, Q}$ follows from [S2, Proposition 2.16]. Next, let

$$
Z=Z(K):=\left\{z \in \mathbb{C}^{N}: V_{K, Q}(z) \leq M=M(K):=\left\|V_{K, Q}\right\|_{K}\right\} .
$$

Then $V_{K, Q}(z)=V_{Z}(z)+M$ for $z \in \mathbb{C}^{N} \backslash Z$ since both functions are maximal outside $Z$ and agree on $\partial Z$ (cf. $[\mathrm{K}]$ ). Thus the function $u(z):=$ $\max \left[0, V_{K, Q}(z)-M\right]$ belongs to $L$ and is equal to 0 at all points of $Z$; hence $u=V_{Z}$ on all of $\mathbb{C}^{N}$. In particular, $V_{Z}$ is continuous; this implies the continuity of $\varrho_{K, Q}=\varrho_{Z}+M$ ([S1, Proposition 2.3(ii)]) and the existence of the directional limits.

We would like to adapt the arguments used to prove Proposition 4.3 and Corollary 4.4 to study $\varrho_{V^{(n)}}=\varrho_{V_{K}^{(n)}}$ for $n=2,3, \ldots$ To this end, we need a modified version of Corollary 4.2 , which in turn requires a generalization of Lemma 4.1.

Lemma 4.7. Fix a positive integer $n \geq 2$ and $m>1$. There exist constants $C_{1}, C_{2}$ and $R$ depending on $n$ and $C_{3}$ depending on $n$ and $m$ such that for each nonpolar set $X \subset \Delta_{m}:=\{t \in \mathbb{C}:|t|<m\}$ and all nonconstant polynomials $p: \mathbb{C}^{N} \rightarrow \mathbb{C}$ of degree at most $n$,

$$
\begin{aligned}
\left|V_{X}^{*}(p(z+\eta))-V_{X}^{*}(p(z))\right| \leq & C_{1}|\eta|\|p\|_{\bar{B}} / \log |z|+C_{2}|\eta|^{2}\|p\|_{\bar{B}}^{2} /(\log |z|)^{2} \\
& +C_{3}|\eta|\|p\|_{\bar{B}} /\left(|z|^{n-1}(\log |z|)^{2}\right)
\end{aligned}
$$

for all $|\eta| \leq 1$ and all $|z| \geq R$ with $|p(z)| \geq \log |z| \cdot|z|^{n-1}$. 
Proof. Let $p: \mathbb{C}^{N} \rightarrow \mathbb{C}$ be a polynomial of degree $n$. We have the following Cauchy estimate for $p$ :

$$
\left|D^{\alpha} p(z)\right| \leq \frac{\alpha !\|p\|_{P(z, 1 / n)}}{(1 / n)^{|\alpha|}}
$$

where $P(z, 1 / n)$ is the polydisc $\Delta\left(z_{1}, 1 / n\right) \times \ldots \times \Delta\left(z_{N}, 1 / n\right)$.

If $z \in \bar{B}:=\bar{B}(0,1)$ and $w \in P(z, 1 / n)$ then $w \in B(0,1+\sqrt{N} / n)$, hence, by the Bernstein-Walsh inequality (cf. [S2]),

$$
\|p\|_{P(z, 1 / n)} \leq\|p\|_{\bar{B}}(1+\sqrt{N} / n)^{n} .
$$

Putting these estimates together for an arbitrary $z \in \bar{B}$, and using the fact that $\alpha ! \leq n^{|\alpha|}$, we get

$$
\left\|D^{\alpha} p\right\|_{\bar{B}} \leq n^{2|\alpha|}(1+\sqrt{N} / n)^{n}\|p\|_{\bar{B}}
$$

for all multi-indices $\alpha$; hence, again by the Bernstein-Walsh inequality, $\left|D^{\alpha} p(z)\right| \leq n^{2|\alpha|}(1+\sqrt{N} / n)^{n}|z|^{n-|\alpha|}\|p\|_{\bar{B}}, \quad$ where $z \in \mathbb{C}^{N}$ with $|z| \geq 1$.

Now fix a polynomial $p$ with $\|p\|_{\bar{B}}=1$ and write

$$
p(z+\eta)=p(z)+\eta \nabla p(z)+O\left(|\eta|^{2}\right) p_{2}(z)
$$

where $p_{2}(z)$ involves at least second-order partial derivatives of $p$. From the above inequality, we have

$|p(z+\eta)-p(z)| \leq\left[n^{2} N|\eta||z|^{n-1}+c_{2}|\eta|^{2}|z|^{n-2}+\ldots+c_{n}|\eta|^{n}\right](1+\sqrt{N} / n)^{n}\|p\|_{\bar{B}}$ for each such $p$ where $c_{2}=c_{2}(n, N), \ldots, c_{n}=c_{n}(n, N)$ are independent of $p$. Thus, for $|z|>R=R(n)$ and $|\eta| \leq 1$,

$$
|p(z+\eta)-p(z)| \leq A n^{2}|\eta||z|^{n-1}\|p\|_{\bar{B}}
$$

where $A=A(n, N)$. Hence, if we write $p(z+\eta)=p(z)+q_{\eta}(z):=p(z)+q(z)$, then (4.5) can be written as

$$
|q(z)| \leq A n^{2}|\eta||z|^{n-1}\|p\|_{\bar{B}} .
$$

To estimate

$$
\left|V_{X}^{*}(p(z+\eta))-V_{X}^{*}(p(z))\right|=\left|\int_{X} \log \frac{|p(z+\eta)-t|}{|p(z)-t|} d \mu_{X}(t)\right|,
$$

we estimate

$$
\begin{aligned}
\log \frac{|p(z+\eta)-t|}{|p(z)-t|} & =\log \frac{|p(z)+q(z)-t|}{|p(z)-t|} \\
& =\log \frac{|p(z)+q(z)|}{|p(z)|}+\log \frac{|p(z)+q(z)-t||p(z)|}{|p(z)-t||p(z)+q(z)|} .
\end{aligned}
$$


First, for $|z| \geq R$ with $|p(z)| \geq \log |z| \cdot|z|^{n-1}$ and $|\eta| \leq 1$, using (4.6) we have

Thus

$$
\frac{|q(z)|}{|p(z)|} \leq \frac{A n^{2}|\eta|\|p\|_{\bar{B}}}{\log |z|} .
$$

$$
\log \left|1+\frac{q(z)}{p(z)}\right| \leq \frac{A n^{2}|\eta|\|p\|_{\bar{B}}}{\log |z|}+O\left(\frac{|\eta|^{2}\|p\|_{\bar{B}}^{2}}{(\log |z|)^{2}}\right)
$$

for such $z, \eta$. Then

Hence

$$
\begin{aligned}
\frac{|p(z)+q(z)-t||p(z)|}{|p(z)-t||p(z)+q(z)|} & \leq \frac{\left|p(z)^{2}+p(z) q(z)-t p(z)\right|}{\left|p(z)^{2}+p(z) q(z)-t p(z)\right|-|t q(z)|} \\
& =1+O\left(\frac{|t q(z)|}{\left|p(z)^{2}+p(z) q(z)-t p(z)\right|}\right) \\
& =1+O\left(\frac{|t q(z)|}{|p(z)|^{2}}\right) .
\end{aligned}
$$

$$
\log \left(\frac{|p(z)+q(z)-t||p(z)|}{|p(z)-t||p(z)+q(z)|}\right)=O\left(\frac{|t q(z)|}{|p(z)|^{2}}\right)=O\left(\frac{m|\eta|\|p\|_{\bar{B}}}{|z|^{n-1}(\log |z|)^{2}}\right) .
$$

The "big- $O$ " terms in these estimates depend on $\|p\|_{\bar{B}}$; note, however, that the points $z$ for which these estimates hold depend on $p(z)$ (since we require $\left.|p(z)| \geq \log |z| \cdot|z|^{n-1}\right)$.

Now we modify Corollary 4.2. For $m>1$, we define

$$
\begin{aligned}
u_{m}(z) & =u_{m, K}^{(n)}(z) \\
& :=\sup \left\{\frac{1}{n} V_{p(K)}(p(z)): p=H_{n}+H_{n-1}+\ldots,\left\|H_{n}\right\|_{\bar{B}}=1,\|p\|_{\bar{B}} \leq m\right\} .
\end{aligned}
$$

Note that $\left\{u_{m}\right\}_{m=2,3, \ldots}$ increases pointwise to $V:=V^{(n)}$ on all of $\mathbb{C}^{N} ;$ moreover, if each $u_{m}$ is continuous, then by Dini's theorem, $u_{m} \rightarrow V$ uniformly on compact subsets of $\mathbb{C}^{N}$.

Corollary 4.8. Fix a positive integer $n \geq 2$ and $m>1$. Let $K \subset B$ be a regular compact set. There exists $C \geq 1$ depending on $n, m$ and $K$ such that for any $\varepsilon>0$, there exists $R=R(m, n, K, \varepsilon)>1$ with

$$
\left|u_{m}(z+\eta)-u_{m}(z)\right| \leq C|\eta| / \log |z|+\varepsilon \quad \text { for all }|\eta| \leq 1,|z|>R \text {. }
$$

Proof. We first make a remark on the use of Lemma 4.7 for $p$ of degree $n$ with $\|p\|_{\bar{B}} \leq m$. By Lemma 4.7 , since $C_{1}, C_{2}$ and $C_{3}$ are independent of $p$, and $\|p\|_{\bar{B}} \leq m$, we can choose $R^{\prime}=R^{\prime}(n, m)$ sufficiently large and $C=C(n, m)$ so that

$$
\left|V_{p(K)}(p(z+\eta))-V_{p(K)}(p(z))\right| \leq C|\eta| / \log |z|
$$

for all such $p$ if $|\eta| \leq 1,|z| \geq R^{\prime}$, and $|p(z)| \geq \log |z| \cdot|z|^{n-1}$. 
Since $K$ is nonpluripolar, $V \in L^{+}$(cf. Section 2) and hence $u_{m} \in L^{+}$; thus, there exist $C_{1}^{\prime}, C_{2}^{\prime}$ with

$$
\log ^{+}|z|+C_{1}^{\prime} \leq u_{m}(z) \leq \log ^{+}|z|+C_{2}^{\prime}
$$

in all of $\mathbb{C}^{N}$; indeed, we can take $C_{1}^{\prime}=0$ since $K \subset B$. Now, to begin the actual proof of Corollary 4.8, given $\varepsilon>0$, we choose $R>R^{\prime}$ so that $\log R \gg \max \left[\left|C_{1}^{\prime}\right|,\left|C_{2}^{\prime}\right|, \varepsilon\right]$. Given $|z|>R$, choose $p=p_{z}=H_{n}+H_{n-1}+\ldots$ with $\left\|H_{n}\right\|_{\bar{B}}=1$ and $\|p\|_{\bar{B}} \leq m$ such that

$$
u_{m}(z) \geq \frac{1}{n} V_{p(K)}(p(z)) \geq u_{m}(z)-\varepsilon .
$$

Using Lemma 3.0, for some $a>0$ we have $\operatorname{cap}(p(K)) \geq a$ if $\left\|H_{n}\right\|_{\bar{B}}=1$. Thus there exists $c^{\prime}=c^{\prime}(a)>0$ such that

$$
\log ^{+}|w| \leq V_{p(K)}(w) \leq \log ^{+}|w|+c^{\prime}
$$

for all $p$ with $\left\|H_{n}\right\|_{\bar{B}}=1$ and all $w \in \mathbb{C}$; combining (4.7), (4.8) and (4.9) for $p=p_{z}$ at the point $z$ we obtain

$$
\log ^{+}|p(z)|+\left(c^{\prime}-n C_{1}^{\prime}\right) \geq n \log ^{+}|z|-n \varepsilon .
$$

By the choice of $R$, we have

$$
|p(z)| \geq \frac{|z|^{n}}{e^{n\left(\varepsilon-C_{1}^{\prime}\right)+c^{\prime}}} \geq b_{1}|z|^{n}
$$

where $b_{1}=b_{1}(a):=1 / e^{n\left(1-C_{1}^{\prime}\right)+c^{\prime}}$ (we may assume $\varepsilon<1$ ). Note $c^{\prime}>0$ and recall we can take $C_{1}^{\prime}=0$ since $K \subset B$; hence $0<b_{1}=e^{-\left(n+c^{\prime}\right)}<1$.

Now given $|\eta| \leq 1,|z|>R$, choose $p=p_{z}$ so that (4.8) holds and $p_{\eta}=\widetilde{H}_{n}+\widetilde{H}_{n-1}+\ldots$ with $\left\|\widetilde{H}_{n}\right\|_{\bar{B}}=1$ and $\left\|p_{\eta}\right\|_{\bar{B}} \leq m$ so that

$$
u_{m}(z+\eta) \geq \frac{1}{n} V_{p_{\eta}(K)}\left(p_{\eta}(z+\eta)\right) \geq u_{m}(z+\eta)-\varepsilon
$$

and $\left|p_{\eta}(z+\eta)\right| \geq b_{1}|z+\eta|^{n} \geq b|z|^{n}$ where $b=b\left(b_{1}\right)$ (as in the proof of Corollary 4.2). Since $b_{1}$ (and hence $b$ ) depends only on $K$ (from (4.7)) and $a$ (from (4.9)), we can assume from the beginning that $b_{1} R \geq \log R$ so that $(+)$ is valid. Using (4.8), (4.10) and the fact that

$$
\frac{1}{n} V_{p_{\eta}(K)}\left(p_{\eta}(z)\right) \leq u_{m}(z), \quad \frac{1}{n} V_{p(K)}(p(z+\eta)) \leq u_{m}(z+\eta),
$$

we obtain

$$
\begin{aligned}
\left|u_{m}(z+\eta)-u_{m}(z)\right| & \leq \frac{1}{n} \max \left[\left|V_{p_{\eta}(K)}\left(p_{\eta}(z+\eta)\right)-V_{p_{\eta}(K)}\left(p_{\eta}(z)\right)\right|,\right. \\
& \left.\left|V_{p(K)}(p(z+\eta))-V_{p(K)}(p(z))\right|\right]+\varepsilon \\
& \leq \frac{1}{n} C|\eta| / \log |z|+\varepsilon
\end{aligned}
$$


Finally, the analogue of Proposition 4.3 follows by applying Corollary 4.8.

Corollary 4.9. For $K \subset B$ a regular compact set, for $n=2,3, \ldots$, and $m>1$, define

$u_{m}(z):=\sup \left\{\frac{1}{n} V_{p(K)}(p(z)): p=H_{n}+H_{n-1}+\ldots,\left\|H_{n}\right\|_{\bar{B}}=1,\|p\|_{\bar{B}} \leq m\right\}$.

Then for each $\alpha \in \partial B$, the directional limit

$$
\lim _{|\lambda| \rightarrow \infty}\left[u_{m}(\lambda \alpha)-\log |\lambda|\right]=\varrho_{u_{m}}(\alpha)
$$

exists.

REMARK. It seems likely that Corollary 4.9 is valid for $V^{(n)}, n=$ $2,3, \ldots$, but we have been unable to verify this.

5. Final remarks. We give an explicit example of a compact set $K$ in $\mathbb{C}^{N}, N>1$, such that $\varrho_{K}$ is not continuous. Note from Corollary 4.6 that $K$ cannot be regular. Indeed, we construct such an example with $K$ circled, i.e., $z \in K$ if and only if $e^{i t} z \in K$. Let

$$
H:=\left\{u \in L: u(\lambda z)=u(z)+\log |\lambda| \text { for } \lambda \in \mathbb{C}, z \in \mathbb{C}^{N}\right\}
$$

be the log-homogeneous psh functions. For $K$ circled,

$$
\begin{aligned}
& V_{K}(z)=\max [0, \sup \{u(z): u \in H, u \leq 0 \text { on } K\}] \\
& =\max \left[0, \sup \left\{\frac{1}{\operatorname{deg} p} \log |p(z)|: p \text { homogeneous polynomial, }\|p\|_{K} \leq 1\right\}\right]
\end{aligned}
$$

moreover, we have the following.

Lemma 5.1. Let $K \subset \mathbb{C}^{N}$ be compact, circled, and nonpluripolar. Then $V_{K}^{*}(z)=\max \left[0, \varrho_{K}(z)\right]$.

Proof. This follows from the above formula for $V_{K}$ and the definition of $\varrho_{K}$. If $V_{K}^{*}(z)>0$, then

$$
\begin{aligned}
\varrho_{K}(z) & :=\limsup _{|\lambda| \rightarrow \infty}\left[V_{K}^{*}(\lambda z)-\log |\lambda|\right] \\
& =\limsup _{|\lambda| \rightarrow \infty}\left[V_{K}^{*}(z)+\log |\lambda|-\log |\lambda|\right]=V_{K}^{*}(z) .
\end{aligned}
$$

Thus $\varrho_{K} \in H$ and $\varrho_{K}(z)=V_{K}^{*}(z)$ if $V_{K}^{*}(z)>0$; hence $\left\{z \in \mathbb{C}^{N}: \varrho_{K}(z) \leq 0\right\}$ differs from $K$ by at most a pluripolar set and the result follows.

The following example is due to Cegrell $[\mathrm{C}]$; we elaborate on the details. Let $\left\{a_{j}\right\}$ be a countable dense sequence of points in the unit circle and let $\left\{\alpha_{j}\right\}$ be a sequence of positive numbers with $\sum_{j} \alpha_{j}<\infty$. We can reorder the $\left\{a_{j}\right\}$ and choose the $\left\{\alpha_{j}\right\}$ accordingly so that, in addition, $\sum_{j} \alpha_{j} \log \left|1-a_{j}\right|>-\infty$. For example, for $n=1,2, \ldots$ and $j=$ 
$2^{n}+1, \ldots, 2^{n+1}$, we can take $\alpha_{j}=2^{-2 n} ;$ and we take $a_{j}, j=2^{n}+1, \ldots, 2^{n+1}$, to be the $2^{n}$-roots of unity (omitting 1 and repeating any other). Define

$$
g\left(z_{1}, z_{2}\right):=\exp \left\{\sum_{j} \alpha_{j} \log \left|z_{1}-a_{j} z_{2}\right|\right\} .
$$

Then $g$ is discontinuous at all points $\left(z_{1}, z_{2}\right)$ with $\left|z_{1}\right|=\left|z_{2}\right|$ and

$$
\sum_{j} \alpha_{j} \log \left|z_{1}-a_{j} z_{2}\right|>-\infty
$$

Moreover, $\log g \in H\left(\mathbb{C}^{2}\right)$. Next, let

$$
h\left(z_{1}, z_{2}\right):=g\left(z_{1}, z_{2}\right)+\max \left(\left|z_{1}\right|,\left|z_{2}\right|\right) .
$$

Then

$$
\begin{aligned}
N(h) & :=\left\{\left(z_{1}, z_{2}\right) \in \mathbb{C}^{2}: h \text { is discontinuous at }\left(z_{1}, z_{2}\right)\right\} \\
& =\left\{\left(z_{1}, z_{2}\right) \in \mathbb{C}^{2}:\left|z_{1}\right|=\left|z_{2}\right| \text { and } \sum_{j} \alpha_{j} \log \left|z_{1}-a_{j} z_{2}\right|>-\infty\right\} .
\end{aligned}
$$

Note that $N(h) \neq \emptyset$ by the assumption that $\sum_{j} \alpha_{j} \log \left|1-a_{j}\right|>-\infty$; indeed,

$$
\left\{\left(r e^{i t}, r e^{i t}\right): 0<r<1,0 \leq t \leq 2 \pi\right\} \subset N(h) .
$$

Now, in $\mathbb{C}^{3}$, we define

$$
\begin{aligned}
W\left(z_{1}, z_{2}, z_{3}\right):= & \exp \left\{\sum_{j} \alpha_{j} \max \left[\log \left|z_{1}-a_{j} z_{2}\right|, \log \left|z_{3}\right|\right]\right\} \\
& +\max \left(\left|z_{1}\right|,\left|z_{2}\right|,\left|z_{3}\right|\right) .
\end{aligned}
$$

Then $\log W \in H\left(\mathbb{C}^{3}\right)$ and from the discussion on $N(h)$, we see that $N(W):=\left\{\left(z_{1}, z_{2}, z_{3}\right) \in \mathbb{C}^{3}: W\right.$ is discontinuous at $\left.\left(z_{1}, z_{2}, z_{3}\right)\right\} \subset\left\{z_{3}=0\right\}$ and

$$
\left\{\left(z_{1}, z_{2}, z_{3}\right) \in \mathbb{C}^{3}:\left(z_{1}, z_{2}\right) \in N(h), z_{3}=0\right\} \subset N(W)
$$

so that $N(W)$ is nonempty and pluripolar. Let

$$
D:=\left\{\left(z_{1}, z_{2}, z_{3}\right) \in \mathbb{C}^{3}: W\left(z_{1}, z_{2}, z_{3}\right)<1\right\} .
$$

Then by a result of Siciak [S3], $K:=\bar{D}$ is a compact, circled, and nonpluripolar subset of $\mathbb{C}^{3}$ with

$$
V_{K}^{*}=\max [0, \log W]=\max \left[0, \varrho_{K}\right] .
$$

More generally, the above argument is valid for any $W \in H\left(\mathbb{C}^{N}\right)$ with $W(z) \geq c|z|, c>0$ and $N(W) \neq \emptyset$ to yield a compact set $K=\bar{D}$ such that $\varrho_{K}$ is not continuous; here, $D=\left\{z \in \mathbb{C}^{N}: W(z)<1\right\}$.

We end the paper with the following relationship on the asymptotic behavior of $\varrho_{V^{(n)}}$ for $K \subset B$ a regular compact set. 
TheORem 5.2. For $K \subset B$ a regular compact set,

$$
\lim _{n \rightarrow \infty} \varrho_{V(n)}(z)=\varrho_{K}(z)
$$

for q.e. $z \in \mathbb{C}^{N}$.

Proof. Note for $n=1,2, \ldots$ that

$$
\begin{aligned}
V^{(n)}(z) & =\sup \left\{\frac{1}{\operatorname{deg} p} V_{p(K)}(p(z)): 1 \leq \operatorname{deg} p \leq n\right\} \\
& \geq \sup \left\{\frac{1}{\operatorname{deg} p} \log ^{+} \frac{|p(z)|}{\|p\|_{K}}: 1 \leq \operatorname{deg} p \leq n\right\} \\
& \geq \sup \left\{\frac{1}{|\alpha|} \log \frac{\left|p_{\alpha}(z)\right|}{\left\|p_{\alpha}\right\|_{K}}: 1 \leq|\alpha|=\operatorname{deg} p_{\alpha} \leq n\right\}
\end{aligned}
$$

where $\left\{p_{\alpha}\right\}$ is any sequence of polynomials with $1 \leq \operatorname{deg} p_{\alpha} \leq n$. Thus we have

$$
\varrho_{V^{(n)}}(z) \geq \sup \left\{\frac{1}{|\alpha|} \log \frac{\left|\widehat{p}_{\alpha}(z)\right|}{\left\|p_{\alpha}\right\|_{K}}: 1 \leq|\alpha|=\operatorname{deg} p_{\alpha} \leq n\right\}
$$

where $\widehat{p}_{\alpha}$ denotes the top degree homogeneous part of $p_{\alpha}$. On the other hand, if we take a family of Chebyshev-type polynomials $\left\{Q_{\alpha}\right\}$ as in, e.g., [Bl, Theorem 2.3], then

$$
\left[\limsup _{|\alpha| \rightarrow \infty} \frac{1}{|\alpha|} \log \frac{\left|\widehat{Q}_{\alpha}(z)\right|}{\left\|Q_{\alpha}\right\|_{K}}\right]^{*}=\varrho_{K}(z) .
$$

Thus

$$
\lim _{n \rightarrow \infty}\left[\sup \frac{1}{|\alpha|} \log \frac{\left|\widehat{p}_{\alpha}(z)\right|}{\left\|p_{\alpha}\right\|_{K}}: 1 \leq|\alpha|=\operatorname{deg} p_{\alpha} \leq n\right]=\varrho_{K}(z) \quad \text { q.e., }
$$

which, together with (5.1), shows that $\lim _{n \rightarrow \infty} \varrho_{V^{(n)}}(z)=\varrho_{K}(z)$ q.e.

REMARK. It is not always true that if $u_{n}, u \in L$ and $u_{n}$ increases pointwise to $u$, then $\varrho_{u_{n}}$ increases q.e. to $\varrho_{u}$ (as a simple example, take $\left.u_{n}(z)=(1-1 / n) \log |z|\right)$. A necessary and sufficient condition that this occurs, even with $u_{n}, u \in L^{+}$, is given in [BT, Theorem 6.6]; a condition that is admittedly very difficult to verify in practice.

6. Open questions. 1. Compute $\mu_{K}^{(n)}:=\left(d d^{c} V_{K}^{(n)}\right)^{N}$ for $K$ regular. Does $\mu_{K}^{(n)}$ have compact support?

2. Let

$$
K=\left\{(x, y) \in \mathbb{R}^{2}: x, y \geq 0, x+y \leq 1\right\} .
$$

In $[\mathrm{BCL}]$, it was shown that $V_{K}^{(1)} \neq V_{K}$. Compute $V_{K}^{(1)}$ explicitly. 
3. Compute $\mu_{K}^{(n)}:=\left(d d^{c} V_{K}^{(n)}\right)^{2}$ for the set

$$
K=\left\{(x, y) \in \mathbb{R}^{2}: x, y \geq 0, x+y \leq 1\right\} .
$$

Does $\mu_{K}^{(n)}$ have compact support?

\section{References}

[BT] E. Bedford and B. A. Taylor, Plurisubharmonic functions with logarithmic singularities, Ann. Inst. Fourier (Grenoble) 38 (1988), 133-171.

[Bl] T. Bloom, Some applications of the Robin function to multivariable approximation theory, J. Approx. Theory 92 (1998), 1-21.

[BCL] L. Bos, J.-P. Calvi and N. Levenberg, On the Siciak extremal function for real compact convex sets in $\mathbb{R}^{N}$, Ark. Mat. 39 (2001), 245-262.

[C] U. Cegrell, On the spectrum of $A(\Omega)$ and $H^{\infty}(\Omega)$, Ann. Polon. Math. 58 (1993), 193-199.

[K] M. Klimek, Pluripotential Theory, Clarendon Press, Oxford, 1991.

[R] L. I. Ronkin, Introduction to the Theory of Entire Functions of Several Variables, Transl. Math. Monogr. 44, Amer. Math. Soc., Providence, 1974.

[S1] J. Siciak, A remark on Tchebysheff polynomials in $\mathbb{C}^{N}$, Univ. Iagel. Acta Math. 35 (1997), 37-45.

[S2] - Extremal plurisubharmonic functions in $\mathbb{C}^{N}$, Ann. Polon. Math. 39 (1981), $175-211$.

[S3] —, Balanced domains of holomorphy of type $H^{\infty}$, Mat. Vesnik 37 (1985), 134-144.

Department of Mathematics

University of Toronto

Toronto, ON, Canada M5S 3G3

E-mail: bloom@math.toronto.edu
University of Auckland

Private Bag 92019

Auckland, New Zealand E-mail: nlevenbe@indiana.edu sione@math.auckland.edu 